\title{
Emphysematous pyelonephritis: Our experience in managing these cases
}

\author{
Fatih Uruc, MD; Ozgur Haki Yuksel, MD; Aytac Sahin, MD; Ahmet Urkmez, MD; Caglar Yildirim, MD; \\ Ayhan Verit, MD
}

Fatih Sultan Mehmet Research and Training Hospital, Istanbul

Cite as: Can Urol Assoc J 2015;9 (7-8):E480-3. http://dx.doi.org/10.5489/cuaj.2828 Published online July 17, 2015.

\section{Abstract}

Introduction: Emphysematous pyelonephritis (EPN) is a rare acute necrotising infection of renal parenchyma. We discuss clinical details and treatment strategies of 8 patients with EPN followed at our clinic.

Methods: We retrospectively reviewed the clinical, laboratory, radiological findings and treatment modalities of 8 patients with EPN followed at our urology clinic between 2012 and 2015.

Results: The mean patient age (female: 5; male: 3) was 62 (range: 51-82) years. Based on computed tomographic findings, EPN was classified as class $1(\mathrm{n}=3)$, class $2(\mathrm{n}=3)$ and class $3 \mathrm{a}$ $(\mathrm{n}=2)$. All patients had fever, flank pain, nausea, and vomiting. Five patients had type 2 diabetes mellitus and 3 diabetic patients also had renal stones. Escherichia coli $(\mathrm{n}=6)$, Klebsiella species $(n=1)$, and Proteus species $(n=1)$ were grown in urine cultures. All patients had unilateral involvement. Increased white blood cell counts, sedimentation rate, and C-reactive protein levels were detected in all cases. In addition to medical treatment, 2 patients underwent a nephrostomy catheter placement and another 2 patients underwent nephrectomy upon deterioration of her general health state. After achieving clinical stabilization with medical treatment, 1 patient underwent endoscopic ureteral stone treatment. The remaining 3 cases were treated only with antibiotherapy. All patients were discharged with clinical cure.

Conclusion: Mortality rates of EPN are gradually decreasing. Preservation of renal reserve is possible due to early diagnosis, appropriate antibiotherapy, and drainage.

\section{Introduction}

Emphysematous pyelonephritis (EPN) is a rare, but highly mortal necrotizing and suppurative disease. It is generally seen in diabetic patients and characterized by gas formation in intrarenal or perirenal regions. ${ }^{1}$ Its overall mortal- ity rate ranges between $19 \%$ and $43 \% .^{2}$ Its most frequent clinical manifestations are fever, flank pain, and pyuria. In addition, non-specific abdominal pain, nausea, vomiting, loss of conscious, shock, costovertebral angle tenderness, dysuria, local crepitation and pneumaturia are also seen. ${ }^{3-5}$ Standard EPN treatment includes parenteral antibiotherapy and percutaneous surgical drainage. ${ }^{3}$

In diabetic patients with symptoms of renal dysfunction and sepsis, a high degree of suspicion should be entertained for EPN and necessary radiological techniques should be performed. Establishing an early diagnosis decreases mortality rates. We discuss clinical details and treatment strategies of 8 patients with EPN followed at our clinic, and review the relevant literature.

\section{Methods}

We retrospectively reviewed the clinical, laboratory, radiological findings and treatment modalities of 8 patients with EPN followed at our urology clinic between 2012 and 2015. Age, clinical, laboratory and radiological findings, duration of treatment and treatment strategies were analyzed. AII patients were classified based on their computed tomographic (CT) data and their predisposing factors were analyzed. Patients were classified according to the Clasification of Huang and Tseng based on CT. ${ }^{2}$ All patients were followed up for an average of 6 months after termination of their treatment.

\section{Results}

The mean patient age (female: 5, male: 3) was 62 (range: 51-82) years. Based on CT findings, we classified their EPN as class $1(n=3)$, class $2(n=3)$, and class $3 a(n=2)$ (Table 1). All patients had fever, flank pain, nausea, and vomiting. Five patients had type 2 diabetes mellitus and 3 of them 


$\begin{aligned} & \text { Table 1. Huang and Tseng Classification of patients with } \\
& \text { EPN }{ }^{2}\end{aligned}$
\begin{tabular}{lr} 
Class I & Gas in the collecting system only \\
Class II & Parenchymal gas only \\
Class IIIA & Extension of gas into perinephric space \\
Class IIIB & Extension of gas into pararenal space \\
Class IV & EPN in a solitary kidney, or bilateral disease \\
\hline CT: computed tomography; EPN: emphysematous pyelonephritis.
\end{tabular}

had also renal stones. Five of 6 patients with stone disease had also grade I-II hydronephrosis. A non-diabetic patient with obstructive ureteral stone had also grade III hydroureteronephrosis. Escherichia coli $(\mathrm{n}=6)$, Klebsiella species $(n=1)$, and Proteus species $(n=1)$ were grown in urine cultures. All patients had unilateral involvement. Right$(n=5)$ and left-sided $(n=3)$ involvement were detected. Increased white blood cell (WBC) counts $\left(20-30 \times 10^{3} / \mathrm{mm}^{3}\right)$, sedimentation rates $(50-100 \mathrm{~mm} / \mathrm{h})$, and C-reactive protein levels $(12-28 \mathrm{mg} / \mathrm{L})$ levels were noted in all cases.

At the time of diagnosis, thrombocytopenia was detected in 2 diabetic patients. In our diabetic patients, glycemic levels were not regulated. In our 2 diabetic patients with ureteral stones, electrolyte and acid-base imbalance were found. In 4 of our 8 patients, renal function was impaired and creatinine levels were between 1.5 and $2.3 \mathrm{mg} / \mathrm{dL}$. Four of 8 patients with electrolyte imbalance and general condition impairment were admitted to the intensive care unit. Two of 4 patients were diabetic and underwent nephrectomy. After surgery, clinical conditions rapidly improved and patients were transferred to the urology division. In addition to medical treatment, a nephrostomy tube was implanted in 2 patients (Fig. 1) and another 2 patients whose general health state deteriorated despite medical therapy underwent radical nephrectomy (Fig. 2). Nephrectomy was performed by lumbothomy incision and extraperitoneal approach to avoid polluting the peritoneal cavity. In 1 patient after achieving clinical stabilization with medical treatment, endoscopic ureteral stone therapy was applied (Fig. 3). The remaining 3 patients were treated only with antibiotherapy. At the time of diagnosis, empirical treatment was initiated with ceftriaxone and metronidazole and medical treatment was revised based on antibacterial susceptibility test results and in consultation with our Department of Infectious Diseases. All patients were discharged with cure.

At the 6-month follow up, 4 of our 8 patients, with creatinine levels in the normal range at diagnosis, had no significant changes in their creatinine values. Creatinine levels of 2 patients who underwent nephrectomy ranged from 1.8 to $2.5 \mathrm{mg} / \mathrm{dL}$. The last remaining 2 patients' creatinine values decreased to normal limits after treatment. The limitations of this study are its retrospective design and lack of aftertreatment renal function testing.

\section{Discussion}

EPN is a necrotizing infection of renal parenchyma and surrounding tissues. It is characterized by gas formation in renal parenchyma, collecting system or perinephric tissues. Kelly and MacCallum reported the first clinical description of the disease in $1898 .{ }^{6}$ However the term emphysematous pyelonephritis was first used over half a century later by Schultz and Klorfein. ${ }^{7}$ Primary microorganisms, which cause EPN, can be normally found in the gastrointestinal and urinary systems. Most frequently, Escherichia coli is identified; many other microorganisms, including klebsiella, proteus and streptococcus, can be isolated. ${ }^{8}$ Even in rare cases, candida and anaerobic microorganisms have also been reported. ${ }^{9,10}$ However, Clostridium has never been isolated

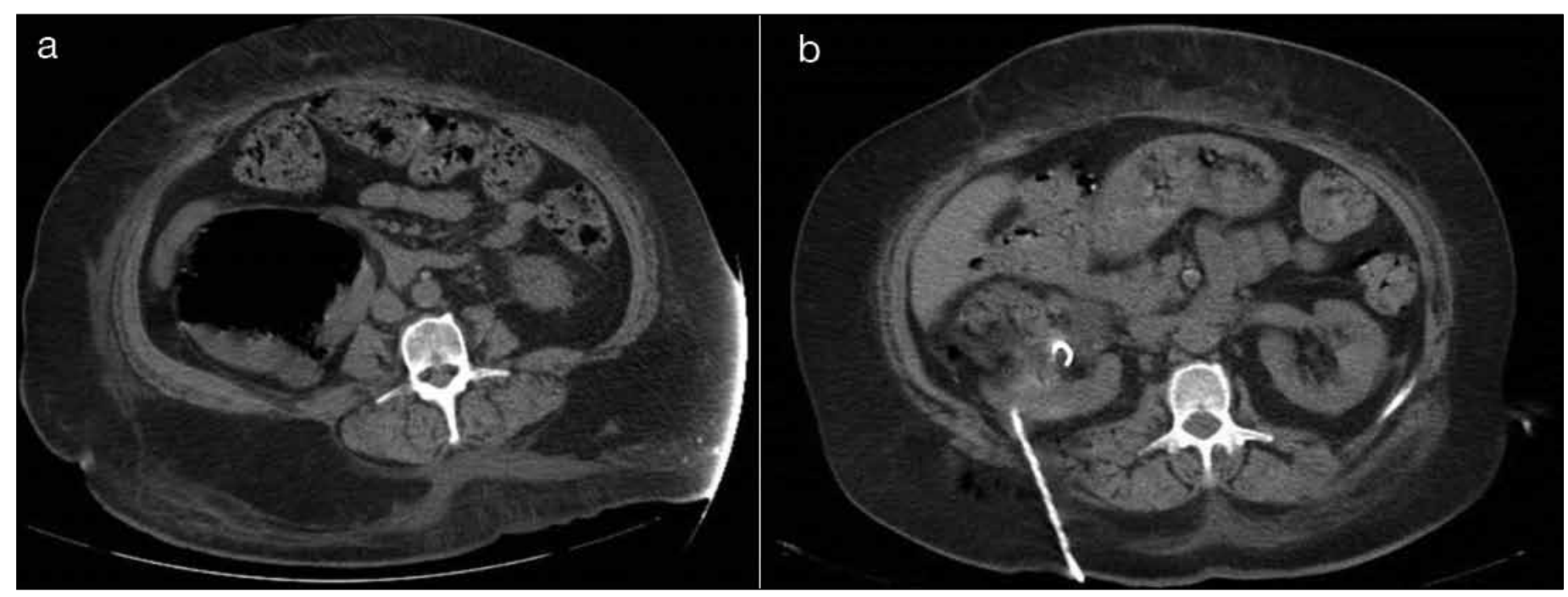

Fig. 1. Patient treated with percutaneous nephrostomy who had diagnosis of class 2 emphysematous pyelonephritis. 


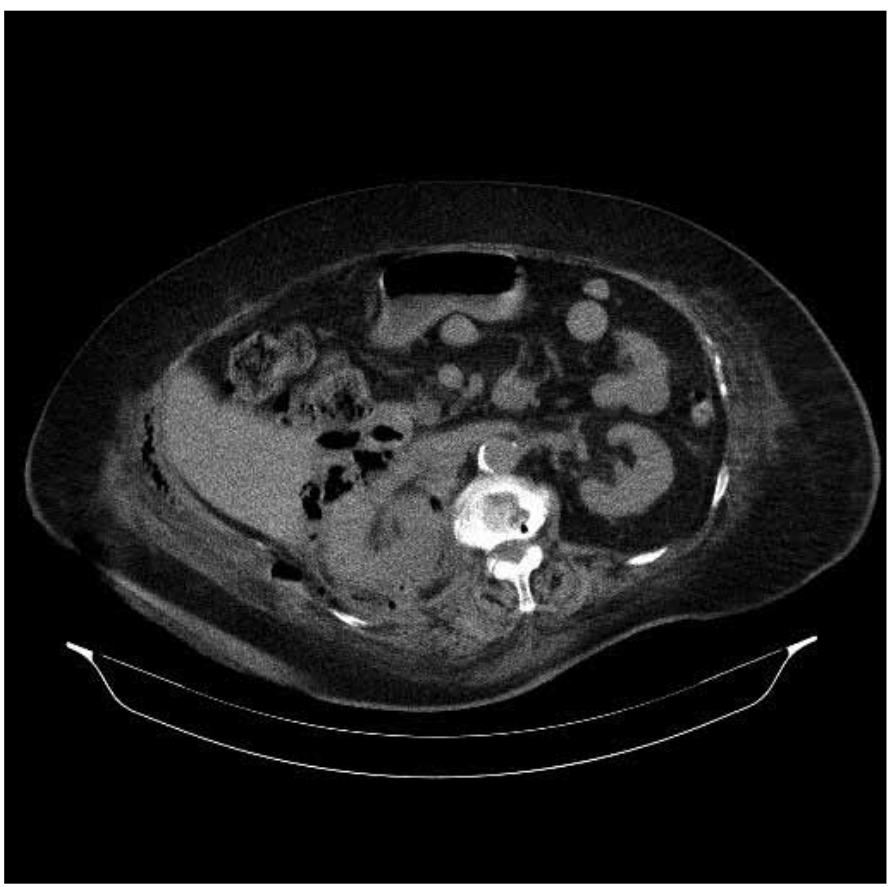

Fig. 2. Patient with a diagnosis of class 3-a emphysematous pyelonephritis treated with nephrectomy.

in this type of infection, though it is a well-known gas forming bacteria. ${ }^{11}$ More than $90 \%$ of the patients are diabetic and it is seen 6 times more frequently in women than men. ${ }^{4}$ Females are more likely to suffer from EPN, with only one exception: males undergoing renal transplantation are more likely to suffer. ${ }^{12}$ Due to a higher concentration of glucose in tissues of patients with diabetes mellitus, hydrogen and carbon dioxide are released through sugar fermentation, which provides a suitable environment for the growth of microorganisms. ${ }^{13}$ In non-diabetic patients generally an obstruction is the underlying cause.

The symptoms, findings and laboratory data of patients with EPN are non-specific and cannot be discriminated from those of upper urinary system infection. Most frequently encountered clinical symptoms include high fever, flank pain, nausea, and vomiting. Crepitation in the lumbar region and pneumaturia can be also observed.

Although there are no specific physical examinations and laboratory findings for EPN, costovertebral angle tenderness, abdominal distension and tenderness, leukocytosis, hyperglycaemia, electrolyte and acid-base imbalance can be detected. Therefore, in suspect cases, diagnosis should be reinforced using radiological methods.

In direct KUB (kidney-ureter-bladder) studies, ipsilateral psoas muscle image is effaced and gas bubbles are observed on renal parenchyma, collecting system or perirenal tissues. Although, ultrasound is the first preferred method in most cases because of its non-invasiveness and easy applicability, it is mostly inadequate in establishing a EPN diagnosis and

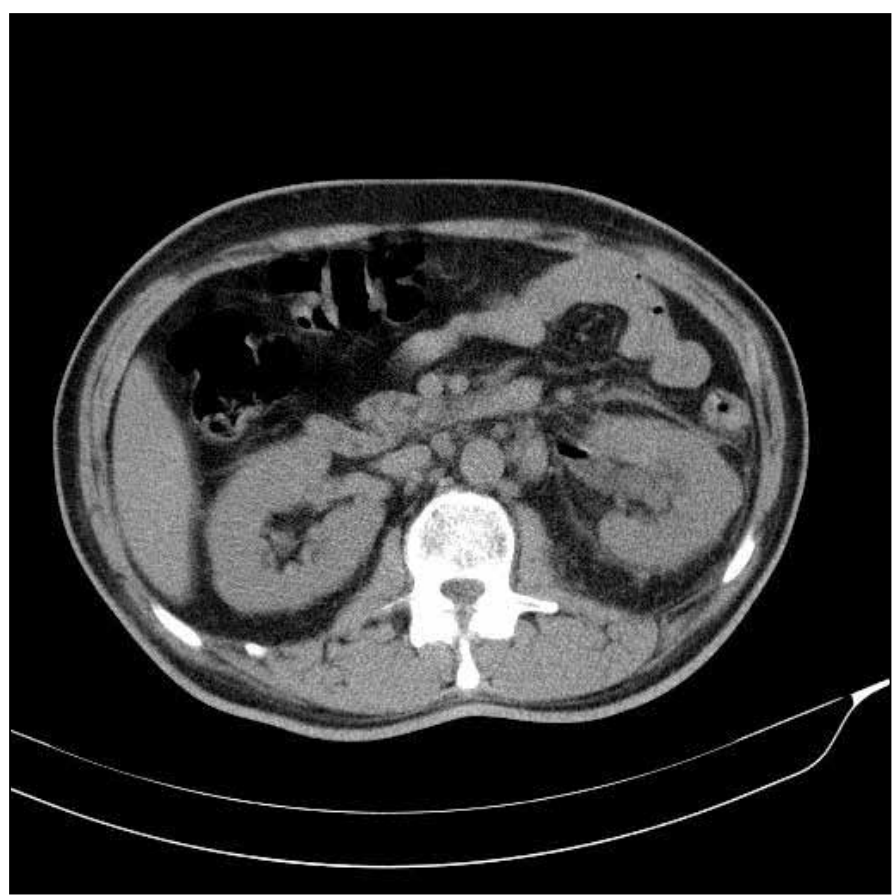

Fig. 3. Patient with a diagnosis of class 1 emphysematous pyelonephriti treated with medical treatment and endoscopic ureteral stone therapy.

in determining its spread. Since these patients are frequently uremic, intravenous pyelography is not preferred. Besides, most of these patients are diabetic, so contrast agents may impair their renal functions. As indicated in previous studies, a CT indisputably demonstrates renal gas and its spread to surrounding tissues. ${ }^{2}$ In addition, CT is also the best tool to use during postoperative follow-up. In our cases, a CT performed after suspicious clinical findings led us to a definitive diagnosis. Gas bubbles on renal parenchyma with clinical findings are specific to, but not patognomic findings of, EPN. Endoscopic procedures, penetrating injuries, and gastrointestinal fistulas can demonstrate similar images on CT. Various clinical and radiological classification methods can be performed in patients with EPN. In our present study, patients were classified according to the Huang and Tseng criteria. Also other classifications exist, such as the one established by Al-Geizawi and colleagues, which depend on the percentage of gas replacing renal parenchyma. ${ }^{14}$

In a literature review, many prognostic factors for mortality were identified; however none of the trials studied a large population. Thrombocytopenia, altered mental status, hyponatremia, severe proteinuria, severe hypoalbuminemia, and acute renal failure at EPN presentation have been associated with a poor outcome. ${ }^{15-17}$ In a meta-analysis, systolic blood pressure less than $90 \mathrm{mmHg}$, serum creatinine levels greater than $2.5 \mathrm{mg} / \mathrm{dL}$, and impairment of consciousness were also associated with increased mortality. ${ }^{18}$ In parallel with these findings, all of our class 1 and 2 patients could be treated with medical therapy and/or drainage; our class 3a patients 
who had adverse predictive factors of thrombocytopenia and impaired consciousness did not respond to these treatment modalities and underwent nephrectomy.

EPN therapy is controversial. Evanoff and colleagues ${ }^{19}$ reviewed EPN cases up to 1987 and detected an average mortality rate of $31 \%$. They reported mortality rates as high as $80 \%$ in patients who received conservative treatment, $60 \%$ in patients who had undergone percutaneous drainage, and $20 \%$ in nephrectomized patients. Shoiker and colleagues ${ }^{4}$ a detected mortality rate of $20 \%$ in their series of emergently nephrectomized 15 cases with EPN following medical stabilization. Management of EPN consists mostly of prevention and treatment of shock, correction of electrolyte imbalance, glycemic regulation, and removal of underlying obstruction (if any). Urine and blood cultures should be obtained and parenteral broad-spectrum antibiotics should be administered. Antibiotherapy should be formulated based on susceptibility test results. Some studies reported success of medical monotherapy in some cases of EPN. ${ }^{20}$ Similarly, Flores and colleagues ${ }^{21}$ had successfully treated acute bilateral EPN using only medical therapy. We also cured 3 patients using only medical therapy without resorting to surgery. We treated all of our patients with parenteral ceftriaxone ( $1 \mathrm{~g}$ bid) and metronidazole (500 $\mathrm{mg}$ bid). However, together with broad-spectrum antibiotics, percutaneous drainage is the most frequently applied treatment modality for EPN. Percutaneous drainage is thought to decrease the burden of infection and spread of infection into surrounding tissues. ${ }^{22}$

\section{Conclusion}

EPN is a rare fatal disease, which requires early diagnosis and initiation of treatment. The most controversial point of this pathological entity is its management. A variety of prognostic factors were specified to determine the appropriate treatment approach of EPN. We think that nephrectomy is an important treatment modality, but there is a trend to maintain conservative treatment regimens by percutaneous techniques. The important clinical issue is to determine the best treatment for each patient.

Competıng interests: The authors declare no competing financial or personal interests.

This paper has been peer-reviewed.

\section{References}

1. Wan YL, Lee TU, Bullard MJ, et al. Acute gas-producing bacterial renal infection: Correlation between imaging finding sand clinical out come. Radiology 1996;198:433-8. http://dx.doi.org/10.1148/ radiology.198.2.8596845

2. Huang JJ, Tseng CC. Emphysematous pyelonephritis: Clinico radiological classification, management, prognosis, and pathogenesis. Arch Intern Med 2000;160:797-5. http://dx.doi.org/10.1001/archinte.160.6.797

3. Pontin AR, Barnes RD. Current management of emphysematous pyelonephritis. Nat Rev Urol 2009;6:2729. http://dx.doi.org/10.1038/nrurol.2009.51

4. Shokeir AA, EL-Azab M, Mohsen T, et al. Emphysematous pyelonephritis: A 15-year experience with 20 cases. Urology 1997;49:343-6. http://dx.doi.org/10.1016/S0090-4295(96)00501-8

5. Kumar VS, Lakshmi AY. Emphysematous pyelonephritis. Indian J Nephrol 2004;14:192-4.

6. Kelly HA, MacCallum WG. Pneumaturia. JAMA 1898;31:375. http://dx.doi.org/10.1001/ jama.1898.92450080001001

7. Schultz EH Jr, Klorfein EH. Emphysematous pyelonephritis. J Urol 1962;87:762-6.

8. Hildebrand $T S$, Nibbe L, Frei $U$, et al. Bilateral emphysematous pyelonephritis caused by Candida infection. Am J Kidney Dis 1999;33:E10. http://dx.doi.org/10.1016/S0272-6386(99)70331-8

9. Johnson JR, Iretion RC, Lipsky BA. Emphysematous pyelonephritis caused by Candida Albicans. J Urol $1986 ; 136: 80-2$

10. Levy AH, Schwinger HN. Gas containing perinephritic abscess. Radiology 1953;60:720. http://dx.doi. org/10.1148/60.5.720

11. Michaeli J, Mogle P, Perberg S, et al. Emphysematous pyelonephritis. J Urol 1984;131:203-8.

12. Tienza A, Hevia M, Merino I, et al. Case of emphysematous pyelonephritis in kidney allograft: Conservative treatment. Can Urol Assoc J 2014;8:E256-9. http://dx.doi.org/10.5489/cuai.1555

13. Stapleton A. Urinary tract infections in patients with diabetes. Am J Med 2002;113:80-4. http://dx.doi. org/10.1016/S0002-9343(02)01062-8

14. Al-Geizawi SM, Farney AC, Rogers J, et al. Renal allograft failure due to emphysematous pyelonephritis: Successful non-operative management and proposed new classification scheme based on literature review. Transpl Infect Dis 2010;12:543-50. http://dx.doi.org/10.1111/i.1399-3062.2010.00538.x

15. Khaira $\mathrm{A}$, Gupta $\mathrm{A}$, Rana DS, et al. Retrospective analysis of clinical profile, prognostic factors and outcomes of 19 patients of emphysematous pyelonephritis. Int Urol Nephrol 2009;41:959-6. http:// dx.doi.org/10.1007/s11255-009-9552-y

16. Kapoor R, Muruganandham K, Gulia AK, et al. Predictive factors for mortality and need for nephrectomy in patients with emphysematous pyelonephritis. BJU Int 2010;105:986-9. http://dx.doi.org/10.1111/ j.1464-410X.2009.08930.x

17. Lu YC, Chiang BJ, Pong YH, et al. Predictors of failure of conservative treatment among patients with emphysematous pyelonephritis. BMC Infect Dis 2014;14:418. http://dx.doi.org/10.1186/14712334- $14-418$

18. Falagas ME, Alexiou VG, Giannopoulou KP, et al. Risk factors for mortality in patients with emphysematous pyelonephritis: A meta-analysis. J Urol 2007;178:880-5. http://dx.doi.org/10.1016/i. juro.2007.05.017

19. Evanoff GV, Thrompson CS, Foley R, et al. Spectrum of gas with in the kidney: Emphysematous pyelonephritis and Emphysematous pyelitis. Am J Med 1987;83:149-4. http://dx.doi.org/10.1016/00029343(87)90511-0

20. Kando T, Okudo H, Suzuki M. A case of emphysematous pyelonephritis improved with conservative therapy- indication for conservative therapy. Hinyokiko Kiyo 2000;46:335-8.

21. Flores $G$, Nellen H, Mayona F. Acute bilateral emphysematous pyelonephritis. Successfully managed by medical therapy alone: A case report and review of the literature. BMJ Nephrol 2002;3:4. http://dx.doi. org/10.1186/1471-2369-3-4

22. Cheng YT, Wang HP, Hsieh HH. Emphysematous pyelonephritis in a renal allograft: Successful treatment with percutaneous drainage and nephrostomy. Clin Transplant 2001;15:364-7. http://dx.doi. org/10.1034/i.1399-0012.2001.150511.x

Correspondence: Dr. Ahmet Urkmez, Fatih Sultan Mehmet Research and Training Hospital, Dept. of Urology, lcerenkoy/Atasehir, Tr-34752 stanbul, Turkey; ahmeturkmez@hotmail.com 